\title{
A Topology Preserving Method for 3-D Non-rigid Brain Image Registration
}

\author{
Vincent Noblet ${ }^{1,2}$, Christian Heinrich ${ }^{1}$, Fabrice Heitz ${ }^{1}$, and \\ Jean-Paul Armspach ${ }^{2}$
}

1 Laboratoire des Sciences de l'Image, de l'Informatique et de la Télédétection, LSIIT, UMR CNRS-ULP 7005, Bd Sébastien Brant, F-67400 Illkirch, France. \{noblet, heinrich, heitz\}@lsiit.u-strasbg.fr

2 Institut de Physique Biologique, Faculté de médecine, UMR CNRS-ULP 7004, 4 Rue Kirschleger, F-67085 Strasbourg Cedex, France.

armspach@ipb.u-strasbg.fr

\begin{abstract}
This paper deals with topology preservation in the framework of 3-D image registration using a multiscale parametric deformation field model. Using a B-spline deformation field yields a polynomial closed form expression for the jacobian. Ensuring positivity of the jacobian guarantees topology preservation. Due to the intricate form of the jacobian in the $3-\mathrm{D}$ case, we have to resort to interval analysis techniques to solve the corresponding optimization problem.
\end{abstract}

\section{Introduction}

Inter-subject or atlas-subject non-rigid brain image registration has received considerable attention during the last decade 1]. The purpose is to estimate long-distance and highly nonlinear deformations corresponding to anatomical variability between individuals without violating topology as anatomical structures, for non-pathological cases, have the same topology across individuals. In order to enforce topology preservation, the transformation should be one-to-one, which is ensured by the positivity of the jacobian. We are thus facing a constrained optimization problem. This work is the 3 -D extension of [2], which only tackles topology preserving mappings in the 2-D case.

\section{Method}

The transformation between both images is parameterized at different scales, using a decomposition of the deformation vector field $\mathbf{u}$ over a sequence of nested subspaces. The bases of these spaces are generated from dilated and translated versions of a single compactly supported scaling function $\phi$, corresponding to a B-spline function. At resolution $l$, the parameterization of $\mathbf{u}$ is given by:

$$
\mathbf{u}^{l}(x, y, z)=\left[\begin{array}{l}
u_{x}^{l}(x, y, z) \\
u_{y}^{l}(x, y, z) \\
u_{z}^{l}(x, y, z)
\end{array}\right]=\left[\begin{array}{l}
\sum_{i, j, k} a_{x ; i, j, k}^{l} \phi_{i, j, k}^{l}(x, y, z) \\
\sum_{i, j, k} a_{y ; i, j, k}^{l} \phi_{i, j, k}^{l}(x, y, z) \\
\sum_{i, j, k} a_{z ; i, j, k}^{l} \phi_{i, j, k}^{l}(x, y, z)
\end{array}\right]
$$


where $\phi_{i, j, k}^{l}(x, y, z)=2^{\frac{3 l}{2}} \phi\left(2^{l} x-i\right) \phi\left(2^{l} y-j\right) \phi\left(2^{l} z-k\right)$. Let $\Omega_{i, j, k}^{l}$ the support of $\phi_{i, j, k}^{l}$. The parameters of this model are estimated by minimizing the quadratic cost function $E(\mathbf{u})=\int_{\Omega}\left|I_{1}(\mathbf{s})-I_{2}(\mathbf{s}+\mathbf{u}(\mathbf{s}))\right|^{2} \mathbf{d s}$, where $\Omega$ is the bounded domain defined by the images and $I_{2}$ is the floating image to be mapped onto image $I_{1}$.

The jacobian $J$ of the transformation must be positive on the whole domain $\Omega$ to preserve topology. The parameters $\left[a_{x ; i, j, k}^{l}, a_{y ; i, j, k}^{l}, a_{z ; i, j, k}^{l}\right]$ of $\mathbf{u}$ are updated blockwise along a direction $\mathbf{d}$ in a Gauss-Seidel scheme. On the corresponding updated block $\Omega_{i, j, k}^{l}$, the jacobian has a closed form polynomial expression of the space variables and the displacement step $\delta$. As its expression is linear in $\delta$, the maximum (resp. minimum) of $J$ on $\Omega_{i, j, k}^{l}$ is a convex (resp. concave) function of $\delta$. An admissible set of steps $\left[0, \delta_{\max }\right]$ is determined using interval analysis techniques [4] to minimize $E$ while satisfying the positivity constraint.

\section{Results}

To highlight the contribution of topology preservation, we present on Fig. 1 the matching between two 3-D $128^{3} \mathrm{MR}$ brain images of different patients, focusing our attention on segmented ventricles. These registrations were performed up to scale $l=5$ (corresponding to 89373 parameters) with a computational burden lower than 30 minutes on a PC workstation $(2.4 \mathrm{GHz})$.
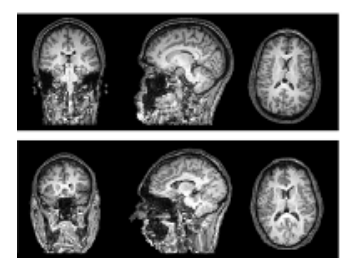

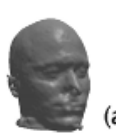

(a)

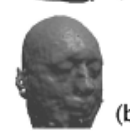

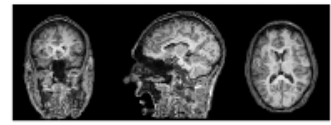

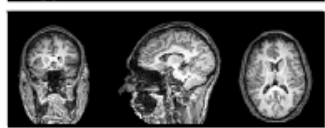

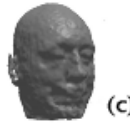
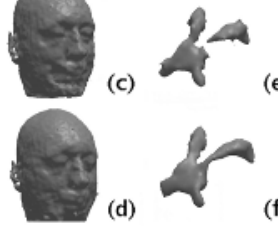

(c)

(e)

(d)

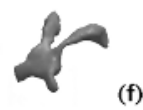

Fig. 1. Non-rigid matching between two different patients: (a) source image; (b) target image; (c) result of matching without any constraint; (d) result of matching with the positivity constraint $J>0$; (e) segmented registered ventricle without any constraint (notice the tearing); (f) segmented registered ventricle with the positivity constraint.

\section{References}

1. J.B.A Maintz and M.A. Viergever: A survey of medical image registration. Medical Image Analysis, 2(1):1-36,1998.

2. O. Musse, F. Heitz, J.P. Armspach: Topology preserving deformable image matching using constrained hierarchical parametric models. IEEE Transactions on Image Processing, 10(7):1081-1093,2001.

3. C. Heinrich, V. Noblet, F. Heitz, J.P. Armspach: 3D deformable image registration: a topology preservation scheme based on hierarchical multiscale deformation models and interval analysis optimization. Preprint, LSIIT UMR CNRS-ULP 7005, Strasbourg, France.

4. L. Jaulin, M. Kieffer, O. Dirit, E. Walter: Applied interval analysis, Springer, 2001. 